\title{
Seroprevalencia de sifilis en mujeres embarazadas en San Luis Potosí
}

\author{
Daniel E N oyola, MC, ${ }^{(1,2)} 0$ ctavio Malacara-Alfaro, MC, ${ }^{(1,3)}$ Victoria Lima-Rogel, MC, ${ }^{(1,3)}$ \\ A braham Torres-Montes, MC. ${ }^{(1,3)}$
}

\section{Noyola DE, Malacara-Alfaro O, Lima-Rogel V, Torres-MontesA. \\ Seroprevalencia de sífilis en mujeres embarazadas en San Luis Potosí. Salud Publica Mex 2006;48:151-154.}

\section{Resumen}

Objetivo. D eterminar la sero prevalencia de sífilis en mujeres embarazadas. Material y métodos. Encuesta seroepidemiológica en 1857 mujeres que acudieron para la atención del parto a un hospital general de la ciudad de San Luis Potosí. Resultados. Se diagnosticó sífilis en cinco (0.27\%) mujeres al momento del parto. Los factores maternos asociados con una probabilidad superior de presentar sífilis incluyeron mayor edad materna, mayor número de embarazos previos y vivir en unión libre con su pareja. Conclusiones El número de recién nacidos expuestos a sífilis durante el embarazo, en la ciudad de San Luis Potosí, está subestimado. Los resultados de este estudio sustentan la necesidad de identificar, al momento del parto, a madres infectadas con sifilis.

Palabras clave: sífilis; sífilis congénita; prevención y control; México

\author{
Noyola DE, Malacara-Alfaro O, Lima-Rogel V, \\ Torres-Montes A. \\ Seroprevalence of syphilis in pregnant \\ women in San Luis Potosí. \\ Salud Publica Mex 2006;48:151-154.
}

\section{A bstract}

Objective.To determine the seroprevalence of syphilis in pregnant women. Methods. A seroepidemiologic survey was conducted in 1857 women giving birth at a general hospital in the city of San Luis Potosi, Mexico. Results Five women $(0.27 \%)$ were diagnosed with syphilis at the time of delivery. Maternal factors associated with a greater likeliho od of syphilis included older age, a higher number of pregnancies and living out of wedlock. Conclusions The number of new borns exposed to syphilis during pregnancy in San Luis Potosi is underestimated. The results of this study support the need to identify syphilis in infected mothers at the time of delivery.

Key words: syphilis; congenital syphilis; prevention $\&$ control; Mexico a sífilis congénita (SC) afecta a recién nacidos en todo el mundo. Durante las últimas décadas se ha notificado un aumento de casos de SC en varios países. ${ }^{1-3}$ Se calcula que en América Latina 330 mil mujeres embarazadas tienen una prueba positiva para sífilis y no reciben tratamiento. ${ }^{4}$ La prevalencia notificada de sífilis en mujeres embarazadas varía en las distintas naciones, de tal manera que va desde $0.4 \%$ en Panamá a $6.2 \%$ en El Salvador. De estos embarazos, se calcula que nacen 110 mil niños con SC y otros 110 mil resul-

(1) Departamento de Pediatría, Hospital Central D r. Ignacio Morones Prieto.

(2) Departamento de Microbiología. Facultad de Medicina, Universidad Autónoma de San Luis Potosí, México.

(3) Pediatría, Facultad de Medicina, Universidad Autónoma de San Luis Potosí, San Luis Potosí, México.

Fecha de recibido: 9 de marzo de 2005 - Fecha de aprobado: 28 de octubre de 2005

Solicitud de sobretiros: Dr. Daniel E. N oyola. D epartamento de Microbiología, Facultad de Medicina, Universidad Autónoma de San Luis Potosí. Avenida Venustiano Carranza 2405. Col. Los Filtros. 78210 San Luis Potosí, S.L.P. México. Correo electrónico:dnoyola@ uaslp.mx 
tan en aborto espontáneo. Dada la importancia de este problema, la Organización Panamericana de la Salud ha propuesto estrategias regionales para la detección y tratamiento de la sífilis durante el embarazo. ${ }^{5}$

El número de casos notificados de SC en México es bajo, lo que podría hacer pensar a la comunidad médica que no se trata de un problema de importancia. ${ }^{6}$ Entre 2002 y 2004 se informaron de 100 a 117 casos anuales de sífilis congénita en México. Tan sólo el estado de Chihuahua notificó $42.5 \%$ de todos los casos del país durante esos tres años; las entidades que colindan con Estados Unidos de América, en conjunto, señalaron las dos terceras partes de todos los casos. ${ }^{7,8}$

En México, el tamizaje para detectar a pacientes con sífilis durante el embarazo está indicado por ley. ${ }^{9}$ Sin embargo, no todos los médicos que atienden a mujeres embarazadas tienen acceso a las pruebas de laboratorio. Además, algunas mujeres no acuden a control prenatal de forma regular. Durante 2002, en el Hospital Central Dr. Ignacio Morones Prieto de la ciudad de San Luis Potosí, se diagnosticó la presencia de SC en tres niños. En ese año hubo sólo un caso adicional de SC notificado por las demás instituciones a los servicios de salud del estado. Con base en esta observación, se planteó la hipótesis de que los casos de sífilis durante el embarazo podrían pasar desapercibidos. Por lo tanto, se realizó una encuesta serológica para determinar la seroprevalencia de anticuerpos contra sífilis en mujeres embarazadas que acudieron al Hospital Central Dr. Ignacio Morones Prieto para atención del parto.

\section{Material y métodos}

\section{Tipo de estudio. Encuesta seroepidemiológica}

Sujetos. Se llevó a cabo una encuesta seroepidemiológica con todas aquellas mujeres embarazadas que acudieron para la atención del parto al Hospital Central Dr. Ignacio Morones Prieto, del 29 de abril al 28 de noviembre de 2003. A todas se les tomó una muestra sanguínea $(3 \mathrm{ml})$ al ingresar a la sala de partos. El Comité de Investigación y Ética del hospital aprobó el protocolo de estudio. Las características demográficas de las madres y de los recién nacidos se registraron en formas estandarizadas. Los embarazos con productos gemelares se registraron como un solo evento y se utilizaron las características del primer gemelo para las comparaciones entre recién nacidos.

Pruebas serológicas. Se realizó la prueba de VDRL utilizando reactivos comerciales (VDRL Latex, Bio-Rad, Marnes-la-Coquette, Francia). Cuando fueron positi- vos los resultados de la prueba de VDRL, se envió una muestra de suero al Laboratorio Estatal de Salud Pública para efectuar pruebas confirmatorias [fluorescent treponemal antibody absortion -FTA- test; FTA-ABS, Bio-Mérieux, Marcy l'Etoile, Francia]. En estos casos, cuando fue posible, se realizaron pruebas serológicas en el recién nacido y en la pareja de la madre.

En cuatro casos fue imposible realizar la prueba confirmatoria de forma inmediata, ya que las pacientes no se encontraban disponibles para muestreo en ese momento y el volumen remanente de suero era insuficiente para la realización de la misma. En esos casos, se procedió a realizar la prueba de anticuerpos antitreponémicos mediante inmunoensayo enzimático (One Step Syphilis Test; IND Diagnostic Inc., Delta, Canadá) y prueba de RPR con el suero disponible. Posteriormente, en tres de las pacientes se pudo realizar la prueba confirmatoria, mientras que en una de ellas no se pudo obtener la muestra sanguínea para esta prueba.

Tratamiento. Cuando el resultado de la prueba de FTA era negativo, se consideraba que el resultado del VDRL era un falso positivo y que la madre no se encontraba infectada. Si el resultado de la prueba de FTA era positivo, se consideraba confirmado el diagnóstico de sífilis en la madre y ésta era referida para tratamiento. Se evaluó a los recién nacidos de madres diagnosticadas con sífilis, a fin de identificar síntomas, signos, o alteraciones de laboratorio (biometría hemática completa; determinación de transaminasas; examen de líquido cefalorraquídeo y determinación de VDRL en el líquido cefalorraquídeo) consistentes con SC. Los recién nacidos de madres infectadas fueron tratados con penicilina (intravenosa y/o intramuscular) durante 10 días.

Análisis estadístico. Se compararon las características demográficas de las madres con diagnóstico serológico de sífilis con las del resto de las pacientes. Por otra parte, se hizo una comparación entre las variables categóricas utilizando la prueba exacta de Fisher, y las variables continuas con la prueba $t$ de Student o la $\mathrm{U}$ de Mann Whitney. Se consideró como significativo un valor de $p<0.05$, y se utilizó el programa estadístico SPSS versión 8.0.

\section{Resultados}

Durante los siete meses del estudio, 2261 mujeres embarazadas ingresaron al hospital para recibir atención en el parto. Se contó con muestra sanguínea para la determinación de VDRL en 1857 (82.1\%) pacientes, y ésta fue positiva en $12(0.65 \%)$ de ellas. En el cuadro I se muestran los resultados de las pruebas confirmatorias realizadas en las pacientes con resultados 
positivos de VDRL. En un caso no se contó con suero suficiente para la realización de prueba de FTA. Sin embargo, esta paciente tuvo una prueba negativa de anticuerpos antitreponémicos utilizando el inmunoensayo enzimático. Además, se realizó una prueba de RPR en el suero de esta paciente, la cual también fue negativa. Hubo cinco casos confirmados de sífilis mediante la prueba de FTA entre las 12 pacientes con prueba de VDRL positiva;por lo tanto, la prevalencia de anticuerpos contra sífilis en las 1857 mujeres incluidas en la encuesta fue de $0.27 \%$. En el cuadro II se presentan las características de las mujeres con sífilis confirmada y aquellas con pruebas negativas. Se observaron diferencias significativas en la edad materna, número de

\section{Cuadro I}

Resultados de serología para sífilis en mujeres Con PRUeba positiva de VDRL, de su Recién nacido y de sus parejas. San Luis Potosí, México, 2003

\begin{tabular}{|c|c|c|c|c|c|c|c|c|c|}
\hline Caso & $\begin{array}{l}\text { Fecha de } \\
\text { nacimiento }\end{array}$ & $\begin{array}{l}\text { Edad } \\
\text { materna }\end{array}$ & $\begin{array}{l}\text { Título del VDRL } \\
\text { materno }\end{array}$ & $\begin{array}{l}\text { Título de RPR } \\
\text { materno }\end{array}$ & $\begin{array}{l}\text { Resultado de prueba } \\
\text { de FTA-ABS } \\
\text { materna }\end{array}$ & $\begin{array}{c}\text { Anticuerpos } \\
\text { antitreponémicos } \\
\text { maternos }\end{array}$ & $\begin{array}{l}\text { Título de VDRL } \\
\text { del recién } \\
\text { nacido }\end{array}$ & $\begin{array}{l}\text { Título deVDRL } \\
\text { de la pareja }\end{array}$ & $\begin{array}{c}\text { Resultado de prueba } \\
\text { de FTA-ABS } \\
\text { de la pareja }\end{array}$ \\
\hline 1 & $23 / 05 / 03$ & 38 & $1: 2$ & - & Reactivo & - & $1: 1$ & - & - \\
\hline 2 & $13 / 06 / 03$ & 26 & $1: 1$ & - & N egativo & - & N egativo & - & - \\
\hline 3 & $13 / 06 / 03$ & 36 & $1: 2$ & - & Reactivo & - & N egativo & - & - \\
\hline 4 & 09/07/03 & 21 & $1: 8$ & - & Reactivo & - & $1: 2$ & $1: 8$ & Reactivo \\
\hline 5 & $30 / 07 / 03$ & 44 & $1: 2$ & - & Reactivo & - & $1: 2$ & $1: 2$ & N egativo \\
\hline 6 & $21 / 08 / 03$ & 16 & $1: 2$ & - & N egativo & - & $1: 1$ & - & - \\
\hline 7 & $26 / 08 / 03$ & 19 & $1: 1$ & - & N egativo & - & N egativo & N egativo & N egativo \\
\hline 8 & $30 / 08 / 03$ & 19 & $1: 1$ & N egativo & N egativo & N egativo & N egativo & N egativo & N egativo \\
\hline 9 & $30 / 08 / 03$ & 30 & $1: 2$ & $1: 1$ & N egativo & N egativo & N egativo & N egativo & N egativo \\
\hline 10 & $31 / 08 / 03$ & 25 & $1: 2$ & N egativo & - & N egativo & - & - & - \\
\hline 11 & $15 / 09 / 03$ & 27 & $1: 4$ & - & Reactivo & - & $1: 2$ & N egativo & N egativo \\
\hline$\overline{12}$ & $02 / 10 / 03$ & 16 & $1: 2$ & $1: 1$ & N egativo & N egativo & N egativo & - & - \\
\hline
\end{tabular}

\section{Cuadro II}

\section{Características demográficas y clínicas de mujeres embarazadas en Quienes Se realizó serología para sífilis. San Luis Potosí, México, 2003}

\begin{tabular}{|c|c|c|c|}
\hline Características maternas o del recién nacido & $\begin{array}{l}\text { Mujeres sin evidencia serológica de sífilis } \\
\qquad(\mathrm{n}=1852)\end{array}$ & $\begin{array}{l}\text { Mujeres con sífilis } \\
\qquad(n=5)\end{array}$ & $P$ \\
\hline \multicolumn{4}{|l|}{ Características maternas } \\
\hline Edad (mediana) & 23 & 36 & 0.02 \\
\hline N úmero de embarazo (mediana) & 2 & 5 & 0.03 \\
\hline 0 rigen rural & $665 \quad(35.9)^{*}$ & $2(40)$ & 1 \\
\hline \multicolumn{4}{|l|}{ Estado civil } \\
\hline Soltera & $237 \quad(12.9)$ & 0 & \\
\hline Casada & $1208 \quad(65.5)$ & $1(20)$ & 0.002 \\
\hline Unión libre & $398 \quad(21.6)$ & $4(80)$ & \\
\hline Pareja trabaja en Estados U nidos & $\begin{array}{ll}124 & (6.7) \\
\end{array}$ & 0 & 1 \\
\hline Ausencia de control prenatal & $78 \quad(4.2)$ & 0 & 1 \\
\hline \multicolumn{4}{|l|}{ Características del recién nacido } \\
\hline Género masculino & $947 \quad(51.1)$ & $1(20)$ & 0.2 \\
\hline Edad gestacional estimada (media; semanas) & 38.3 & 38.1 & 0.79 \\
\hline Peso (media; gramos) & 3095 & 2780 & 0.32 \\
\hline Talla (media; cm) & 50.3 & 51 & 0.7 \\
\hline Perimetro cefálico (media; cm) & 34 & 33.3 & 0.5 \\
\hline
\end{tabular}


embarazos y el estado civil de las madres con sífilis, en comparación con las madres que tuvieron prueba negativas para sífilis. Las mujeres infectadas eran mayores en edad que aquellas sin infección y, con un número superior de embarazos. Además, vivían en unión libre con más frecuencia que aquéllas sin infección $(R M=14.5$; IC 95\% 1.6-130.3). La evaluación clínica al nacimiento y los exámenes de laboratorio practicados a los recién nacidos de madres con sífilis no revelaron ninguna anormalidad.

\section{Discusión}

El diagnóstico y tratamiento de la sífilis durante el embarazo es importante dada la elevada tasa de transmisión al producto. Los métodos de diagnóstico utilizados para la detección de casos de sífilis incluyen pruebas treponémicas (como el FTA) y no treponémicas (como el VDRL). La prueba de VDRL es útil para programas de tamizaje. Sin embargo, puede dar lugar a resultados falsamente positivos, de tal manera que siempre es necesario confirmar el diagnóstico mediante pruebas treponémicas específicas. La probabilidad de transmisión vertical de sífilis varía de acuerdo con el estadio en que se encuentra la infección de la madre durante el embarazo. La probabilidad de transmisión durante sífilis primaria o secundaria no tratada es de 60 a 90\%; en la sífilis latente temprana, es de $40 \%$, y en la sífilis latente tardía se reduce a menos de $10 \% .^{10,11}$ Los recién nacidos que sufren de infección in utero pueden fallecer o desarrollar secuelas. En México, muchas mujeres reciben atención en unidad médicas que no cuentan con las pruebas serológicas para detección de sífilis. Esto puede resultar en un número importante de infantes expuestos a la sífilis de forma inadvertida durante el embarazo. Durante este estudio se identificó a cinco mujeres con sífilis no tratada que recibieron atención del parto en el Hospital Central. Aunque los recién nacidos de estas madres no mostraron ningún síntoma al nacimiento, es posible que hayan sufrido de infección in utero y, por tanto, que se encontraran en riesgo de desarrollar posteriormente síntomas o secuelas de la infección.

Se observaron algunas diferencias entre las madres infectadas y aquellas en quienes se descartó la presencia de infección. El promedio de edad de las mujeres con sífilis fue mayor que el de que aquellas sin infección. Asimismo, el número de embarazos previos fue superior en las mujeres con infección por sífilis, además de que éstas refirieron vivir en unión libre con más frecuencia que aquellas con serología negativa. Estas observaciones difieren de lo notificado en Estados Unidos de América, en donde las mujeres con mayor riesgo de tener un recién nacido con sífilis con- génita son las madres extranjeras, de grupos minoritarios y que no recibieron atención médica prenatal. ${ }^{12}$ Dado el reducido número de madres identificadas con sífilis en el presente estudio, es imposible definir el perfil de la mujer con riesgo de presentar sífilis en nuestra población

La seroprevalencia de anticuerpos contra sífilis en la población bajo estudio fue de $0.27 \%$. Juárez-Figueroa y colaboradores encontraron reactividad a pruebas de tamizaje y confirmadas con prueba de FTA en $2 \%$ de 200 mujeres durante el embarazo en la ciudad de Cuernavaca, Morelos. ${ }^{6}$ Los resultados que se presentan aquí apoyan las recomendaciones publicadas en septiembre de 2003 e indican la necesidad de realizar pruebas de VDRL en todas las mujeres en el momento del parto en México. ${ }^{13}$

\section{Agradecimientos}

Se agradece a la química farmacobióloga Martha Teresa Torres Rojas y al Dr. Heriberto Lizaola Díaz de León su apoyo durante la ejecución de este proyecto.

\section{Referencias}

1. Hegyi V, D anilla T, H egyi E.Actual trends of the incidence of syphilis and gonorrhoea in the Slovak Republic in the years 1990-6. Sex Transm Infect 1998; 74:376-377.

2. N icoll A, Hamers FF. Are trends in HIV, gonorrhoea, and syphilis worsening in western Europe? BMJ 2002;324:1324-1327.

3. Sánchez PJ, W endel GD. Syphilis in pregnancy. C lin Perinatol 1997;24:71-90.

4.Valderrama J, Zacarías F, Mazin R. Sífilis materna y sífilis congénita en América Latina: un problema grave de solución sencilla. Rev Panam Salud Publica 2004;13:211-217.

5.Valderrama J. Eliminación de la sífilis congénita en A mérica Latina y el Caribe: marco de referencia para su implementación. W ashington, D.C.: O PS, 2005.

6. Juárez-Figueroa LA, Meléndez B, C onde-G onzález CJ. Syphilis at full term pregnancy in women from Cuernavaca, Mexico. Rev Invest $\mathrm{Clin}$ 2001;53:375-377.

7. Secretaría de Salud. Boletín de Epidemiología. Sem 2. México, D.F.: SSA, Dirección General de Epidemiología, 2004.

8. Secretaría de Salud. Boletín de Epidemiología. Sem 2. México, D.F.: SSA, Dirección General de Epidemiología, 2005.

9. Secretaría de Salud. N orma 0 ficial Mexicana N O M-007-SSA2-1993. Atención de la mujer durante el embarazo, parto y puerperio y del recién nacido. Criterios y procedimientos para la prestación del servicio. México, D.F.: SSA, 1993.

10. A merican A cademy of Pediatrics. Syphilis. En: Pickering LK, ed. Red Book:2003 Report of the Committee on Infectious Diseases. 26a. edición. Elk Grove Village (IL):A merican A cademy of Pediatrics, 2003:595-607. 11. Rawstron SA. Treponema pallidum (Syphilis). En: Long SS, Pickering LK, Prober CG, eds. Principles and practice of pediatric infectious diseases. 2a. edición. Philadelphia: Churchill-Livingston, 2003:954-961.

12. Mascola L, Pelosi R, Blount JH, Binkin N J,Alexander CE, Cates W, Jr. Congenital syphilis. Why is it still occurring? JAMA 1984;252:1719-1722. 13. Secretaría de Salud. N orma 0 ficial Mexicana N 0 M-039-SSA2-2002 para la prevención y control de las infecciones de transmisión sexual. 